\title{
Simultaneous Kinetic-Spectrofluorometric Determination of Levodopa and Carbidopa Using Partial Least-Squares Regression
}

\author{
Ariana P. Pagani, Miguel A. Cabezón, and Gabriela A. IbáÑEZ \\ Departamento de Química Analítica, Facultad de Ciencias Bioquímicas y Farmacéuticas, Universidad \\ Nacional de Rosario and Instituto de Química Rosario (IQUIR), Consejo Nacional de Investigaciones \\ Científicas y Técnicas (CONICET), Suipacha 531, Rosario S2002LRK, Argentina
}

\begin{abstract}
A simple and sensitive methodology for the simultaneous determination of levodopa and carbidopa in pharmaceutical samples is described. The method combines the advantages of fluorescence with partial least-squares (PLS) analysis, and requires no previous separation steps. The developed method is based on the oxidation of levodopa and carbidopa by cerium(IV) in a sulfuric acid medium and monitoring the fluorescence of the formed Ce(III) at $\lambda_{\mathrm{exc}}=255 \mathrm{~nm}$ and $\lambda_{\mathrm{em}}=$ $355 \mathrm{~nm}$. PLS uses differences in the reaction rates as a discriminatory parameter, and regresses the data of fluorescence vs. time onto the concentrations of the standards. Eight validation samples and seven commercial tablets were studied, using a nine-sample aqueous calibration set. The analyte recoveries from pharmaceuticals ranged from 98 to $101 \%$ for levodopa and from 100 to $108 \%$ for carbidopa. The results obtained by the developed method were statistically comparable to those obtained with high-performance liquid chromatography.
\end{abstract}

(Received November 21, 2008; Accepted January 6, 2009; Published May 10, 2009)

Parkinson's disease is a progressive neurological disorder that occurs when the brain fails to produce enough dopamine. This condition causes tremor, muscle rigidity and bradykinesia. Dopamine administration is not effective, because it cannot penetrate the blood-brain barrier. The dopamine precursor levodopa (3-(3,4-dihydroxy)-L-phenylalanine) is the therapeutic drug of choice for its treatment. Levodopa can be orally administered, and is enzymatically decarboxylated to dopamine This decarboxylation reaction may occur at some rate before levodopa reaches the brain. For this reason, high doses are needed to provide symptomatic relief, causing adverse reactions, such as nausea and vomiting. In order to enhance its pharmacological effect, levodopa is combined with carbidopa, an inhibitor of decarboxylase activity. Carbidopa ((S)-2-(3,4dihidroxybenzoyl)-2-hydrazinopropionic acid) inhibits the decarboxylation of levodopa in peripheral tissues, thus allowing for a greater amount of levodopa dose to be transported into the central nervous system, increasing its accessibility to the brain. This concomitant administration provides substantial clinical benefits to most patients at the initial stages of disease, with reduced side effects. ${ }^{1-5}$

Several procedures have been described in the literature for the determination of levodopa and carbidopa in pharmaceutical preparations and biological fluids. These methods are: spectrophotometry, ${ }^{6,7}$ gas chromatography, ${ }^{8}$ chemiluminescense, ${ }^{9}$ voltammetric determination, ${ }^{10}$ potentiometry, ${ }^{11}$ radioimmunoassay $^{12}$ and flow injection analysis (FIA). ${ }^{13}$ Their simultaneous determination has been traditionally achieved using high-performance liquid chromatography (HPLC). ${ }^{4,14,15}$ Capillary electrophoresis (CE) has been alternatively used ${ }^{16,17}$ and a method utilizing NMR spectroscopy has also been developed. ${ }^{18}$ In recent years, some spectrophotometic methods have been described in order to resolve both of these

$\dagger$ To whom correspondence should be addressed.

E-mail: ibanez@iquir-conicet.gov.ar compounds: chemometric-assisted kinetic-spectrophotometric methods, ${ }^{2,19}$ a kinetic H-point standard addition method (HPSAM), ${ }^{3}$ and an enzymatic flow-batch system with spectrophotometric detection. ${ }^{20}$ However, as far to our knowledge, only an spectrofluorometric method using double synchronous scans has been reported for the simultaneous determination of levodopa and carbidopa. ${ }^{21}$ It is noteworthy that methods using spectral signals (absorption and excitation and emission spectra) have not been widespread. An explanation for this fact lies in the strong spectral overlapping of both compounds, which in principle precludes the application of multivariate calibration of spectroscopic data for resolution of a mixture of levodopa and carbidopa. Owing to this fact we selected the kinetic profiles as an analytical signal for the simultaneous quantification of levodopa and carbidopa.

As an analytical technique, fluorescence has the basic advantage of considerably greater sensitivity compared with other methodologies. Moreover, kinetic methods are being considered as alternatives for studying mixtures with a small number of analytes to be quantified. It is possible to discriminate among mixtures on the basis of differences in the reaction rates with a common reagent. ${ }^{6} \mathrm{Ce}(\mathrm{IV})$ has been used as an oxidizing agent for the determination of several pharmaceuticals. ${ }^{22-29}$ It can be easily reduced to $\mathrm{Ce}(\mathrm{III})$, which shows a characteristic fluorescence in dilute sulfuric acid. This reaction could be implemented as an expeditious method for the indirect fluorometric determination of several pharmaceutical species that cannot be directly determined by fluorescence. ${ }^{27}$

Multivariate calibration methods are being successfully applied to instrumental data of a variety of sources, mainly spectroscopic, in order to construct predictive models for the determination of mixtures of compounds in several fields. ${ }^{30}$ Recently, chemometric methods based on factor analyses and artificial intelligence have found increasing applications for multicomponent kinetic determinations. ${ }^{2,3,19,30-32}$ Partial leastsquares (PLS) is the most popular regression method for 
multicomponent analysis due to the performance of its calibration models, to the availability of software and to the easiness of its implementation. ${ }^{30}$

The aim of this work is to develop an indirect kineticspectrofluorometric method for the simultaneous determination of levodopa and carbidopa in pharmaceutical preparations. The analytical procedure is based on the oxidation of these drugs by $\mathrm{Ce}(\mathrm{IV})$ in the presence of sulfuric acid, and monitoring of the fluorescence intensity of the formed Ce(III) at $\lambda_{\mathrm{exc}}=255 \mathrm{~nm}$ and $\lambda_{\mathrm{em}}=355 \mathrm{~nm}$. The differences in the obtained kinetic profiles were used to determine mixtures of both compounds by multivariate calibration of the kinetic data, using the PLS-1 model.

The results found upon applying the proposed method have been compared with those obtained by HPLC, used as a method of reference.

It is important to point out that the compounds analyzed in the present paper were previously studied in a completely different context, measuring second-order data (fluorescence emission spectra as a function of the reaction time with cerium), which were then employed as a model to evaluate the performance of the second-order chemometric method multivariate curve resolution-alternating least-squares (MCR-ALS). ${ }^{33}$ In this previous work, the aim was to achieve a so-called second-order advantage (i.e., quantitation of a given analyte in the presence of uncalibrated sample components), under conditions of extreme spectral overlapping (the fluorescence emission spectra). For this latter purpose, carbidopa was considered to be the analyte and levodopa the potential interferent. In the presently discussed work, however, the simultaneous quantitation of both levodopa and carbidopa is described, based on the measurement of firstorder data (kinetic evolution at fixed excitation and emission wavelengths), processed with a first-order multivariate calibration method, such as PLS in the absence of substantial interferents.

\section{Experimental}

\section{Instrumentation and software}

Fluorescence measurements as a function of time were performed on a Varian Cary Eclypse luminescence spectrometer equipped with a $7 \mathrm{~W}$ Xenon pulsed lamp and connected to a PC microcomputer, using $1.00 \mathrm{~cm}$ quartz cells. The instrumental parameters were: excitation and emission slit, $5 \mathrm{~nm}$; $\lambda_{\mathrm{exc}}, 255$ $\mathrm{nm} ; \lambda_{\mathrm{em}}, 355 \mathrm{~nm}$; photomultiplier tube (PMT) sensitivity, $550 \mathrm{mV}$. All measurements were performed in a thermostated cell at $20.0 \pm 0.1^{\circ} \mathrm{C}$ by means of a RM 6 LAUDA thermostatic bath.

Kinetic curves were measured in random order with respect to the analyte concentrations, and those corresponding to the calibration set were recorded on different days with respect to the validation set and commercial samples. Data were saved in the ASCII format, and transferred to a PC Sempron AMD microcomputer for subsequent manipulation by the PLS program. PLS-1 was applied with the program MVC1, ${ }^{34}$ written in MATLAB 6.0, and freely available on the Internet at www. chemometry.com. The program is based on a previously described algorithm. ${ }^{35,36}$ Sigmaplot 9.0 software was used for regression analysis and the treatment of data.

High-perfomance liquid chromatographic procedures were carried out on a Waters (Milford, MA) liquid chromatograph equipped with a 515 Waters high-pressure pump operating at 2.5 $\mathrm{mL} / \mathrm{min}$, a Rheodyne injector with a $20-\mu \mathrm{L}$ sample loop and a variable-wavelength UV-vis detector measuring at $282 \mathrm{~nm}$. A 5$\mu \mathrm{m}$ Zorbax $\mathrm{SB} \mathrm{C}_{18}$ column $(15 \mathrm{~cm} \times 4.6 \mathrm{~mm}$ i.d. $)$ was used at room temperature.

\section{Reagents}

All chemicals used were of analytical reagent grade. For spectrofluorometric measurements the following solutions were employed: 0.1 and $0.25 \mathrm{~mol} \mathrm{~L}^{-1} \mathrm{H}_{2} \mathrm{SO}_{4}$ solutions were prepared from commercial $\mathrm{H}_{2} \mathrm{SO}_{4}$ (Merck, Darmstadt, Germany). Stock solutions of levodopa (Klonal Laboratories, Buenos Aires, Argentina) and carbidopa (Klonal Laboratories), both $600 \mathrm{mg}$ $\mathrm{L}^{-1}$, were prepared by weighting the required amount of the corresponding compounds and, after adding drops of sulfuric acid $0.25 \mathrm{~mol} \mathrm{~L}^{-1}$, dissolving in doubly distilled water. Both solutions were prepared daily. A stock solution of $\mathrm{Ce}(\mathrm{IV})(1.00$ $\times 10^{-3} \mathrm{~mol} \mathrm{~L}^{-1}$ ) was prepared from Ce(IV) sulfate tetrahydrate (Merck) and dissolved in $0.25 \mathrm{~mol} \mathrm{~L}^{-1} \mathrm{H}_{2} \mathrm{SO}_{4}$.

For HPLC determinations, stock solutions of levodopa and carbidopa (10000 and $2000 \mathrm{mg} \mathrm{L}^{-1}$, respectively) were prepared as described above. The mobile phase was prepared following the Pharmacopoeia directions, and consisted in a buffer of 0.1 mol L-1 $\mathrm{NaH}_{2} \mathrm{PO}_{4}\left(\mathrm{NaH}_{2} \mathrm{PO}_{4} \cdot \mathrm{H}_{2} \mathrm{O}\right.$; Merck), adjusted to $\mathrm{pH} 3.0$ with $\mathrm{H}_{3} \mathrm{PO}_{4}$ (Merck). ${ }^{37}$

\section{Calibration and validation sets}

For training the PLS-1 model, a calibration set was constructed by preparing nine calibration samples following a central composite design, with five concentration levels of each analyte (levodopa in the range $0.0-1.20 \mathrm{mg} \mathrm{L}^{-1}$ and carbidopa in the range $0.0-0.55 \mathrm{mg} \mathrm{L}^{-1}$ ). Table 1 gives the composition of the binary mixtures used in the calibration set. All calibration samples were prepared in duplicate and measured in random order.

A validation set with 8 randomized samples was prepared with the concentrations of levodopa and carbidopa, as reported in Table 2. Validation solutions were prepared by mixing appropiate volumes of stock solutions of levodopa and carbidopa in concentrations which were selected at random from their corresponding calibration ranges.

\section{Commercial tablets}

The analyzed pharmaceuticals were purchased from a local drugstore. Commercial tablets of Lebocar 250/25, Lebocar 100/25, Lebocar Ap (Pfizer; Searle Argentina, Buenos Aires, Argentina), Sinemet 250/25, Sinemet 100/25 (Merck Sharp \& Co., Whitehouse Station, NJ), and Prikap 250/25, Prikap100/25 (Elea, Buenos Aires, Argentina) were analyzed. The tablets were processed as follows: 20 tablets were finely powdered, and a suitable amount of the obtained powder was weighed, dissolved in $0.1 \mathrm{~mol} \mathrm{~L}^{-1} \mathrm{H}_{2} \mathrm{SO}_{4}$, sonicated for $30 \mathrm{~min}$ and filtered through a $0.45-\mu \mathrm{m}$ nylon membrane filter. For the spectrofluorometric procedure, the previous solutions were diluted with doubly distilled water to obtain the required concentrations for the assay. The final concentrations and analyte ratios in each test solution were within the corresponding calibration ranges. Each sample solution was prepared in triplicate and measured in random order.

\section{Spectrofluorometric procedure}

The analytes oxidation reaction was carried out directly in a spectrofluorometer cell. In order to obtain the kinetic profiles, calibration, validation and samples solutions were processed as follows: $2.50 \mathrm{~mL}$ of the corresponding solution was added to the quartz cell and thermostated at $20^{\circ} \mathrm{C}$, and $200 \mu \mathrm{L}$ of $\mathrm{Ce}(\mathrm{IV}) 1.00$ $\times 10^{-3} \mathrm{~mol} \mathrm{~L}^{-1}$ in $\mathrm{H}_{2} \mathrm{SO}_{4} 0.25 \mathrm{~mol} \mathrm{~L}^{-1}$ solution was added. Then, the cell was capped and its content was homogenized by turning it upside down. After $10 \mathrm{~s}$, the relative fluorescence of the induced $\mathrm{Ce}$ (III) as a function of time was recorded during $5 \mathrm{~min}$ every $0.008 \mathrm{~min}$ (600 points) at $\lambda_{\mathrm{exc}}=255 \mathrm{~nm}$ and $\lambda_{\mathrm{em}}=355 \mathrm{~nm}$. To avoid interference due to the initial presence of $\mathrm{Ce}(\mathrm{III})$, the 
Table 1 Calibration set for applying the PLS-1 method and the corresponding calibration statistical parameters

\begin{tabular}{|c|c|c|}
\hline $\begin{array}{c}\text { Number of } \\
\text { calibration sample }\end{array}$ & Levodopa/mg L ${ }^{-1}$ & Carbidopa/mg L-1 \\
\hline 1 & 0.276 & 0.432 \\
\hline 2 & 0.948 & 0.119 \\
\hline 3 & 0.612 & 0.551 \\
\hline 4 & 0 & 0.281 \\
\hline 5 & 0.948 & 0.432 \\
\hline 6 & 0.612 & 0 \\
\hline 7 & 0.612 & 0.281 \\
\hline 8 & 1.21 & 0.281 \\
\hline 9 & 0.276 & 0.119 \\
\hline Factor & 2 & 2 \\
\hline Sensor range & $1-200$ & $1-80$ \\
\hline $\mathrm{RMSE}^{\mathrm{a}} / \mathrm{mg} \mathrm{L}^{-1}$ & $3.4 \times 10^{-2}$ & $2.1 \times 10^{-2}$ \\
\hline $\mathrm{REP}^{\mathrm{b}}$ & 5.5 & 7.35 \\
\hline$R^{2 \mathrm{c}}$ & 0.991 & 0.985 \\
\hline Sensitivity $/ \mathrm{mg}^{-1} \mathrm{~L}$ & 415 & 354 \\
\hline$\gamma^{\mathrm{d}} / \mathrm{mg}^{-1} \mathrm{~L}$ & 300 & 300 \\
\hline$(1 / \gamma) / \mathrm{mg} \mathrm{L}^{-1}$ & $3.3 \times 10^{-3}$ & $3.3 \times 10^{-3}$ \\
\hline Selectivity & 0.13 & 0.11 \\
\hline $\mathrm{LOD}^{\mathrm{e}} / \mathrm{mg} \mathrm{L}^{-1}$ & $1 \times 10^{-2}$ & $1 \times 10^{-2}$ \\
\hline
\end{tabular}

a. $\operatorname{RMSE}=\left[(1 / I) \Sigma_{1}^{\mathrm{I}}\left(c_{\text {act }}-c_{\text {pred }}\right)^{2}\right]^{1 / 2}$ where $I$ is the number of calibration samples $(I=18)$.

b. $\mathrm{REP}=(100 \times \mathrm{RMSE}) / \bar{C}$ where $\bar{C}$ is average concentration of the component for the $I$ calibration samples.

c. $R^{2}=1-\Sigma_{1}^{\mathrm{I}}\left(c_{\text {act }}-c_{\text {pred }}\right)^{2} / \Sigma_{1}^{\mathrm{I}}\left(c_{\text {act }}-c_{\text {pred }}\right)^{2}$.

d. $\gamma$ (analytical sensitivity) $=$ sensitivity $/ s_{\mathrm{y}}$, where $s_{\mathrm{y}}$ is instrumental noise

e. $\mathrm{LOD}=3 \times s_{\mathrm{y}} /$ sensitivity.

fluorescence intensity of the Ce(IV) solution was measured daily to obtain an appropriate blank correction: $200 \mu \mathrm{L}$ of $\mathrm{Ce}$ (IV) $1.00 \times 10^{-3} \mathrm{~mol} \mathrm{~L}^{-1}$ in a $\mathrm{H}_{2} \mathrm{SO}_{4} 0.25 \mathrm{~mol} \mathrm{~L}^{-1}$ solution was added to a cell containing $2.50 \mathrm{~mL}$ of doubly distilled water, following the same procedure as described above. The blank signal was subtracted from each measurement.

\section{Chromatographic procedure}

A calibration set was prepared in the range 800 to $1300 \mathrm{mg} \mathrm{L}^{-1}$ for levodopa and 150 to $350 \mathrm{mg} \mathrm{L}^{-1}$ for carbidopa, considering the content of both analytes in the pharmaceuticals tablets. Each pharmaceutical preparation was processed as discussed above (see Commercial tablets). The prepared solutions of tablets $(20.0 \mu \mathrm{L})$ were injected into the HPLC column. Chromatographic separation was accomplished in $600 \mathrm{~s}$ under isocratic conditions, using a buffer phosphate to $\mathrm{pH}=3$ as the mobile phase. The flow rate was maintained at $2.5 \mathrm{~mL} / \mathrm{min}$. The peaks of both analytes were well resolved, with retention times of $130 \mathrm{~s}$ for levodopa and $525 \mathrm{~s}$ for carbidopa. However, since carbidopa is present in pharmaceuticals preparations in significantly smaller concentrations than levodopa, the analytes must be quantitated separately using sample solutions of different concentrations. Each sample solution was injected in triplicate and the concentrations were calculated using the calibration curve.

\section{Partial least-squares (PLS-1)}

PLS is a regression technique for multivariate data, and has been applied to many fields in science with great success. PLS is the most popular regression method for multicomponent analyses, due to its versatility and predictive capacity, and shows several important advantages: 1) it employs full data, a feature
Table 2 Prediction result and statistical analysis for the simultaneous determination of levodopa and carbidopa in validation set using PLS-1 method

\begin{tabular}{llllll}
\hline & \multicolumn{2}{c}{ Levodopa $/ \mathrm{mg} \mathrm{L}^{-1}$} & & \multicolumn{2}{c}{ Carbidopa $\mathrm{a} / \mathrm{mg} \mathrm{L}^{-1}$} \\
\cline { 2 - 3 } \cline { 5 - 6 } & Actual & Predicted & & Actual & Predicted \\
\hline & 0.351 & $0.403(2)$ & & 0.411 & $0.406(2)$ \\
& 0.363 & $0.39(1)$ & & 0.333 & $0.328(9)$ \\
& 0.847 & $0.910(4)$ & & 0.178 & $0.20(2)$ \\
& 0.695 & $0.631(6)$ & & 0.486 & $0.484(1)$ \\
& 0.251 & $0.234(6)$ & & 0.760 & $0.76(4)$ \\
& 0 & N.D. & & 0.473 & $0.51(1)$ \\
& 1.05 & $1.13(1)$ & & 0 & N.D. \\
& 0.929 & $0.950(2)$ & & 0.255 & $0.303(4)$ \\
RMSE/REP & 0.482 & $0.47(1)$ & & 0.532 & $0.574(8)$ \\
$R^{2}$ & $4.7 \times 10^{-2} / 8.2$ & & $3.0 \times 10^{-2} / 9.8$ \\
& \multicolumn{2}{c}{0.981} & & \multicolumn{2}{c}{0.976} \\
\hline
\end{tabular}

N.D.: not detected, concentration of analyte below detection limit a. Experimental standard deviation from duplicate sample analysis between parenthesis.

See Table 1 for RMSE, REP and $R^{2}$.

critical for the resolution of complex multi-analyte mixtures, 2) analytical procedures can be carried out in a short time, usually with no sample clean-up or physical separation, and 3) its calibration models ignore the concentrations of other components, except for a selected analyte in the studied samples. ${ }^{30}$ PLS involves a two-step procedure: (1) calibration, where the relation between vectorial data such as spectra and reference component concentrations is established from a set of standard samples, and (2) prediction, in which the calibration results are employed to estimate the component concentrations in unknown samples. ${ }^{38}$ In the PLS-1 version, all model parameters are optimized for the determination of each analyte at a time. During the model training step, the calibration data are decomposed by an iterative algorithm, which correlates the data with the calibration concentrations using a so-called "inverse" model. ${ }^{35}$ This provides a set of regression coefficients to be applied to a new sample. Before calibration, however, the optimum number of latent variables should be selected in order to avoid overfitting, by applying the leave-one-out crossvalidation method described by Haaland. ${ }^{35}$

\section{Results and Discussion}

\section{Effect of operational parameters}

The kinetic behavior of most chemical species in chemical reactions can be controlled by changing the microenvironment of the reaction. Suitable experimental conditions ( $\mathrm{pH}$, concentration of $\mathrm{Ce}(\mathrm{IV})$ and temperature) were analyzed in order to obtain an adequate analytical signal and to improve the selectivity.

Effect of $\mathrm{Ce}(\mathrm{IV})$ concentration. The influence of the Ce(IV) concentration was studied in the range between $1 \times 10^{-5}$ and $1 \times$ $10^{-4} \mathrm{~mol} \mathrm{~L}^{-1}$. Higher concentrations were not evaluated because of the possibility of a fluorescence inner filter, since $\mathrm{Ce}(\mathrm{IV})$ absorbs at $350 \mathrm{~nm}\left(\lambda_{\max }\right.$ about $\left.320 \mathrm{~nm}\right),{ }^{28}$ the same $\lambda$ at which $\mathrm{Ce}(\mathrm{III})$ emits. The rate of the reaction increases with the concentration of $\mathrm{Ce}(\mathrm{IV})$ up to $2 \times 10^{-5}$ and $3 \times 10^{-5} \mathrm{~mol} \mathrm{~L}^{-1}$ for carbidopa and levodopa, respectively. For values higher than these, the rate of the reaction remains constant (zeroth partial order). This represents an [oxidant]/[analyte] relation of 11 for carbidopa and 14 for levodopa. Therefore, the final 

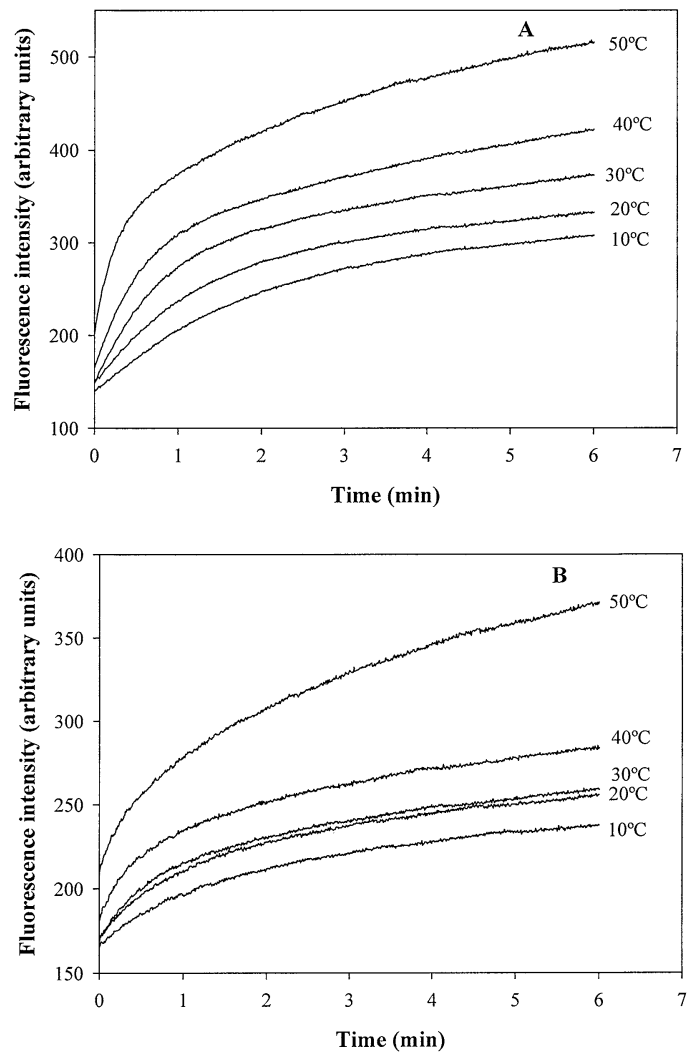

Fig. 1 Effect of temperature on the kinetic profiles of levodopa $\left(0.700 \mathrm{mg} \mathrm{L}^{-1}\right)$ (A) and carbidopa $\left(0.300 \mathrm{mg} \mathrm{L}^{-1}\right)$ (B) systems. Experimental conditions: $\left[\mathrm{H}_{2} \mathrm{SO}_{4}\right], 0.02 \mathrm{~mol} \mathrm{~L}^{-1}$ (given by added cerium solution); [Ce(IV)], $9 \times 10^{-5} \mathrm{~mol} \mathrm{~L}^{-1}$. Instrumental conditions: $\lambda_{\text {exc }}, 255 \mathrm{~nm} ; \lambda_{\text {em }}, 355 \mathrm{~nm}$; band pass, $5 \mathrm{~nm}$; PMT, $550 \mathrm{mV}$.

concentration of $\mathrm{Ce}(\mathrm{IV})$ was fixed at $9 \times 10^{-5} \mathrm{~mol} \mathrm{~L}^{-1}$, while taking into account the calibration range for both analytes. Influence of chemical variables. Selectivity is an important factor to resolve mixtures of analytes, and thus the experimental conditions $(\mathrm{pH}$, temperature) were analyzed and selected to maximize it.

The effect of temperature on the reaction rate of levodopa and carbidopa was studied in the range of $10-50^{\circ} \mathrm{C}$, working at the optimal concentration of $\mathrm{Ce}$ (IV) and at the acidity given by the sulfuric acid contained in the added cerium solution. The kinetic curves at several temperatures are represented in Figs. $1 \mathrm{~A}$ and $1 \mathrm{~B}$. As can be observed, the increase in temperature causes an increase in the reaction rate for both compounds.

The effect of acidity was examined in three different sulfuric acid concentrations: $0.02 \mathrm{~mol} \mathrm{~L}^{-1}$ (given by the added cerium solution), 0.1 and $0.25 \mathrm{~mol} \mathrm{~L}^{-1}$. As shown in Figs. $2 \mathrm{~A}$ and $2 \mathrm{~B}$, the increase in acidity causes a decrease in the reaction rate for both systems.

On the basis of the kinetic profiles, the selectivity under each condition was calculated according to the following equation:

$$
\mathrm{SEL}=\sqrt{\frac{S_{1} \times S_{2}-\left(S_{12}\right)^{2}}{S_{1} \times S_{2}}},
$$

where: $S_{1}=\Sigma p_{1 \mathrm{i}}^{2}, S_{2}=\Sigma p_{2 \mathrm{i}}{ }^{2}$ and $S_{12}=\Sigma p_{1 \mathrm{i}} \times p_{2 \mathrm{i}} ; p_{1 \mathrm{i}}$ is kinetic profile of component 1 at time $i$ and $p_{2 \mathrm{i}}$ is the kinetic profile of component 2 at time $i$.

The values of selectivity obtained when analyzing the different acidity conditions did not significantly differ. The same tendency
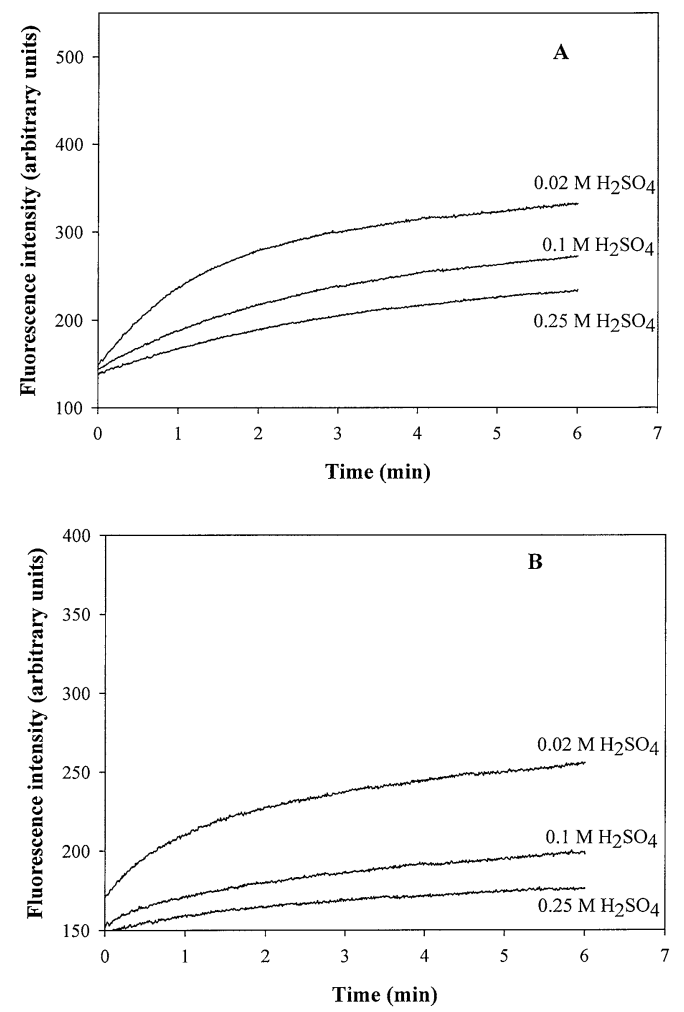

Fig. 2 Effect of acidity on the kinetic profiles of levodopa (0.700 $\left.\mathrm{mg} \mathrm{L}^{-1}\right)(\mathrm{A})$ and carbidopa $\left(0.300 \mathrm{mg} \mathrm{L}^{-1}\right)(\mathrm{B})$ systems. Experimental conditions: $t, 20.0^{\circ} \mathrm{C}$; $[\mathrm{Ce}(\mathrm{IV})], 9 \times 10^{-5} \mathrm{~mol} \mathrm{~L}^{-1}$. Instrumental conditions are the same as in Fig. 1.

was obtained when analyzing the temperature in the range $10-30^{\circ} \mathrm{C}$. However, for values higher than $30^{\circ} \mathrm{C}$, the selectivity decreased. For simplicity and for better control of the experimental variables, and taking into account the intensity of the measured signals, the selected conditions were $20^{\circ} \mathrm{C}$ and the acidity, given by an added cerium solution $\left(0.02 \mathrm{~mol} \mathrm{~L}^{-1} \mathrm{H}_{2} \mathrm{SO}_{4}\right)$.

\section{Spectroscopic and kinetic characteristics}

Levodopa and carbidopa react with Ce(IV) in a sulfuric acid medium to give fluorescent $\mathrm{Ce}(\mathrm{III})$, which presents an emission maximum at $355 \mathrm{~nm}$ when excited at $255 \mathrm{~nm}$. Figure 3 shows excitation and emission spectra corresponding to the carbidopa and levodopa systems induced by oxidation with $\mathrm{Ce}(\mathrm{IV})$, where the obtained maxima correspond to the formed Ce(III). Figure 4 shows the kinetic curves of levodopa and carbidopa during reactions with $\mathrm{Ce}(\mathrm{IV})$ under the selected conditions. Even though the analytes show differences in their reaction rates with $\mathrm{Ce}(\mathrm{IV})$, their kinetic profiles exhibit an important overlapping, making it necessary to use multivariate calibration techniques for resolution. These methods may use the kinetic constants as discriminatory parameters to resolve the mixture of analytes.

\section{Data analysis}

With the aim of determining if the differences of the kinetic constants may discriminate the analytes, a set of calibration and validation samples were prepared and analyzed by PLS-1. In order to build multivariate calibration models for levodopa and carbidopa determination, oxidation curves with Ce(IV) were recorded for all samples. Due to the variability of the blank, the kinetic profiles processed by PLS-1 correspond to those corrected with the appropriate blank. 


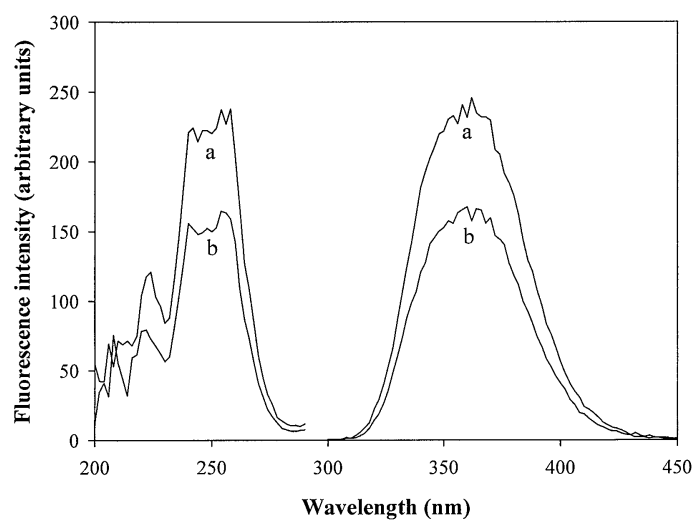

Fig. 3 Excitation and emission spectra corresponding to the oxidation reaction of: (a) levodopa $\left(0.612 \mathrm{mg} \mathrm{L}^{-1}\right)$ and (b) carbidopa $\left(0.281 \mathrm{mg} \mathrm{L}^{-1}\right)$ with $\mathrm{Ce}(\mathrm{IV})$. Experimental conditions: $t, 20.0^{\circ} \mathrm{C}$, [Ce(IV)], $9 \times 10^{-5} \mathrm{~mol} \mathrm{~L}^{-1} ;\left[\mathrm{H}_{2} \mathrm{SO}_{4}\right], 0.02 \mathrm{~mol} \mathrm{~L}^{-1}$. Instrumental conditions are the same as in Fig. 1.

The selected sensor ranges were 1 - 80 for carbidopa and 1 200 for levodopa. Using these sensor ranges, PLS-1 coupled to leave-one-out cross-validation was then performed in order to estimate the number of optimum latent variables in each case, using the Haaland and Thomas criterion. ${ }^{35}$ The optimum number of factors was estimated by computing the ratios $F(A)=$ $\operatorname{PRESS}\left(A<A^{*}\right) / \operatorname{PRESS}(A)\left(\right.$ where $\operatorname{PRESS}=\Sigma\left(c_{\mathrm{i}, \mathrm{act}}-c_{\mathrm{i} \text {,pred }}\right)^{2}$, is the predicted error sum of squares, $c_{\mathrm{i}, \text { act }}$ and $c_{\mathrm{i} \text {,pred }}$ indicate the actual and predicted concentration for the $i$ th sample, $A$ is a trial number of factors and $A^{*}$ corresponds to the minimum PRESS), and selecting the number of factors leading to a probability of less than $75 \%$ that $F>1$. This analysis led to the conclusion that the latter number is two for both calibrations, which allows PLS to explain more than $99 \%$ of the observed variance in the calibration data.

Table 1 summarizes the optimum number of factors for each analyte, the optimal regions used in the calibration and the statistical parameters of calibration: squared correlation coefficient $\left(R^{2}\right)$, relative error of prediction (REP) and root mean square error (RMSE), which provide an indication of the quality of fit of all calibration data. The calculated figures of merit for both analytes (sensitivity, analytical sensitivity $(\gamma)$, its inverse $1 / \gamma$, selectivity and limit of detection (LOD)) are also given in Table 1.

Table 2 gives the prediction results and statistical analysis corresponding to the validation set (binary mixtures). They indicate that the present method is accurate in what concerns binary mixtures, as suggested by the appropriate validation RMSE and REP values.

With the purpose of evaluating the proposed method in real samples, seven commercial tablets containing levodopa and carbidopa were analyzed with the developed method. The obtained values, summarized in Table 3, indicate that the method yields good results concerning the simultaneous determination of levodopa and carbidopa in pharmaceutical samples, with good recoveries: between 98 and 101\% for levodopa and from 100 to $108 \%$ for carbidopa. For the assayed commercial samples, the recoveries are all within the ranges recommended by the British Pharmacopoeia. ${ }^{37}$ The recoveries were calculated while considering the nominal contents declared by the manufacturing laboratories. In order to asses the accuracy of the method, the PLS results were compared with those provided by a reference technique proposed by the Pharmacopoeia, using paired $t$-test statistics at a 95\% confidence level, and revealed no

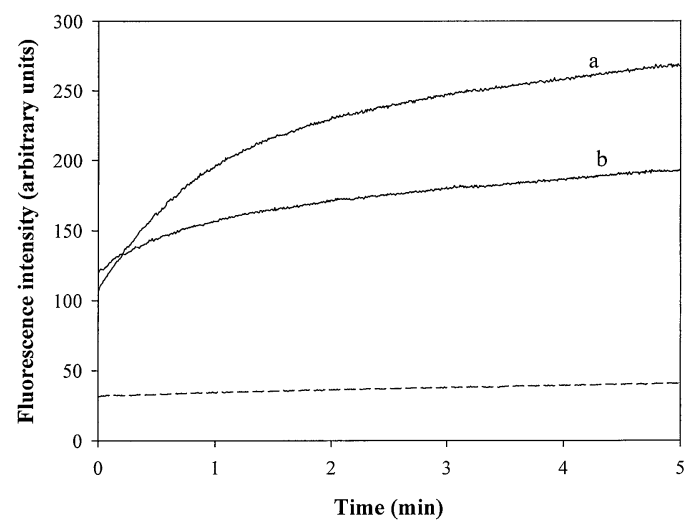

Fig. 4 Kinetic profiles of the oxidation reaction with $\mathrm{Ce}(\mathrm{IV})$ obtained for: (a) levodopa $\left(0.612 \mathrm{mg} \mathrm{L}^{-1}\right)$, (b) carbidopa $(0.281 \mathrm{mg}$ $\mathrm{L}^{-1}$ ) and (c) a blank solution. Experimental and instrumental conditiors are the same as in Fig. 3

significant differences between both methods (Table 3). ${ }^{39}$ Therefore, the excipients present in commercial tablets do not appear to constitute interferences to the developed method.

The proposed method shows an improvement in simplicity and speed with respect to those currently employed for the simultaneous determination of these compounds, such as HPLC and $\mathrm{CE}$, and does not require expensive equipment or the use of organic solvents. Another advantage is that, compared to the existing spectrophotometric methods for the determination of the studied drugs, it is more sensitive,,$^{2,3,19,20}$ and it is similar to the reported spectrofluorometric method. ${ }^{21}$ It can also determine lower concentrations of carbidopa in the presence of higher amounts of levodopa, and thus can be successfully applied to the analysis of tablets that usually contain higher amounts of levodopa with respect to carbidopa.

In conclusion, the proposed method allows for the simultaneous determination of levodopa and carbidopa without any pretreatment (separation or masking) in pharmaceutical samples.

\section{Conclusions}

The simultaneous determination of levodopa and carbidopa in pharmaceutical samples was performed using kinetic profiles of the reaction with $\mathrm{Ce}(\mathrm{IV})$. A first-order mulivariate calibration method (PLS-1) was applied. A validation set of synthetic mixtures as well as pharmaceutical samples were studied. The results discussed above show that PLS-1 is a good alternative to the simultaneous determination of levodopa and carbidopa in pharmaceutical samples based on the difference in the rate of the reaction with a common oxidant, $\mathrm{Ce}(\mathrm{IV})$. The proposed method shows an improvement in simplicity and speed with respect to those currently employed for the simultaneous determination of these compounds, such as HPLC and CE, and is more sensitive than previously reported methods, based on simultaneous spectroscopic determinations. In addition, the results obtained for commercial tablets were statistically comparable to those obtained by the reference Pharmacopoeia method based on HPLC. Additionally, the present study opens the possibility of using the proposed method for the determination of levodopa and carbidopa in biological samples. 
Table 3 Prediction results on commercial samples by applying PLS-1 and comparison with HPLC

\begin{tabular}{|c|c|c|c|c|c|c|c|c|}
\hline \multirow{3}{*}{$\begin{array}{l}\text { Commercial } \\
\text { sample }\end{array}$} & \multicolumn{4}{|c|}{ Levodopaa/mg } & \multicolumn{4}{|c|}{ Carbidopa a/mg } \\
\hline & \multirow{2}{*}{ Reported } & \multicolumn{2}{|c|}{ Found } & \multirow{2}{*}{$t^{\mathrm{b}}$} & \multirow{2}{*}{ Reported } & \multicolumn{2}{|c|}{ Found } & \multirow{2}{*}{$t^{\mathrm{b}}$} \\
\hline & & PLS & HPLC & & & PLS & HPLC & \\
\hline Lebocar 250/25 & 250 & $246(4)[98.4]$ & 250(3) [100] & 1.58 & 25 & $26(3)[104]$ & $24(2)[96]$ & 0.87 \\
\hline Lebocar 100/25 & 100 & $92(2)[99]$ & $101.7(7)[102]$ & 2.21 & 25 & $26(1)[104]$ & $24.8(7)[99.2]$ & 1.95 \\
\hline Lebocar AP & 200 & $200(3)[100]$ & 199(4) [99.5] & 0.13 & 50 & $52(5)[104]$ & $48(1)[96]$ & 1.33 \\
\hline Sinemet $250 / 25$ & 250 & $249(3)$ [99.6] & $252(3)[100.8]$ & 1.31 & 25 & $25(2)[100]$ & $25.0(6)[100]$ & 0 \\
\hline Sinemet $100 / 25$ & 100 & $105(6)[105]$ & $102(4)[102]$ & 0.90 & 25 & $27(2)[108]$ & $25(1)[100]$ & 1.66 \\
\hline Prikap 250/25 & 250 & $253(4)[101]$ & $250(6)[100]$ & 0.73 & 25 & $25(2)[100]$ & $24.5(4)[98]$ & 0.52 \\
\hline Prikap 100/25 & 100 & $99(4)[99]$ & $97(2)[97]$ & 0.62 & 25 & $25.7(6)[103]$ & $24.4(7)[95]$ & 2.64 \\
\hline
\end{tabular}

a. Experimental standard deviation from triplicate sample analysis between parentheses. The recoveries (in brackets) are based on the amount labeled in commercial tablets. b. Calculate values of the statistical $t$ coefficient for the method comparison. The critical values at $95 \%$ confidence level and 4 d.f. is $t(v=4,95 \%)=2.78$.

\section{Acknowledgements}

This research was supported by the Universidad Nacional de Rosario, the Consejo Nacional de Investigaciones Científicas y Técnicas (PIP 5303), Agencia Nacional de Promoción Científica y Tecnológica (Projects PICT06 N²5825 and PAE N 22204). The authors are also thankful to Dr. A. C. Olivieri (Universidad Nacional de Rosario) for his valuable suggestions.

\section{References}

1. L. Zhang, G. Chen, Q. Hu, and Y. Fang, Anal. Chim. Acta, 2000, 431, 287.

2. P. C. Damiani, A. C. Moschetti, A. J. Rovetto, F. Benavente, and A. C. Olivieri, Anal. Chim. Acta, 2005, 543, 192.

3. A. Safavi and M. Tohidi, J. Pharm. Biomed. Anal., 2007, 44, 313.

4. A. Tolokán, I. Klebovich, K. Balogh-Nemes, and G. Horvai, J. Chromatogr., B, 1997, 698, 201.

5. M. Karimi, J. L. Carl, S. Loftin, and J. S. Perlmutter, J. Chromatogr., B, 2006, 836, 120.

6. J. Coello, S. Maspoch, and N. Villegas, Talanta, 2000, 53, 627.

7. P. Nagaraja, R. A. Vasantha, and K. R. Sumitha, J. Pharm. Biomed. Anal., 2001, 25, 417.

8. P. S. Doshi and D. J. Edwards, J. Chromatogr., 1981, 210, 505.

9. M. L. Yang, L. Q. Li, M. L. Feng, and J. R. Lu, Yaowu Fenxi Zazhi, 1998, 18, 41 .

10. M. F. Bergamini, A. Santos, N. R. Stradiotto, and M. V. B. Zanoni, J. Pharm. Biomed. Anal., 2005, 39, 54.

11. S. S. Badawy, Y. M. Issa, and A. S. Tag-Eldin, Electroanalysis, 1996, 8, 1060.

12. C. J. Waugh, I. F. Gow, C. R. W. Edwards, and B. B. Williams, Biochem. Soc. Trans., 1989, 17, 163.

13. M. Pistonesi, M. E. Centurión, B. S. Fernández Band, P. C. Damiani, and A. C. Olivieri, J. Pharm. Biomed. Anal., 2004, 36, 541 .

14. K. A. Sagar and M. R. Smyth, J. Pharm. Biomed. Anal., 2000, 22, 613.

15. M. Karimi, J. L. Carl, S. Loftin, and J. S. Perlmutter, J. Chromtogr., B, 2006, 836, 120.

16. L. Zhang, G. Chen, Q. Hu, and Y. Fang, Anal. Chim. Acta, 2001, 431, 287.

17. P. T. T. Ha, A. Van-Schepdael, T. Hauta-Aho, E. Roets, and J. Hoogmartesn, Electrophoresis, 2002, 23, 3404.
18. Z. Talebpour, S. Haghgoo, and M. Shamsipur, Anal. Chim. Acta, 2004, 506, 97.

19. M. Chamsaz, A. Safavi, and J. Fadaee, Anal. Chim. Acta, 2007, 603, 140.

20. M. Grünhut, M. E. Centurión, W. D. Fragoso, L. F Almeida, M. C. U. de Araújo, and B. S. Fernández Band, Talanta, 2008, 75, 950.

21. W. H. Kim, M. M. Karim, and S. H. Lee, Anal. Chim. Acta, 2008, 619, 2.

22. P. Y. Khashaba, J. Pharm. Biomed. Anal., 2002, 27, 923.

23. F. A. Mohamed, H. A. Mohamed, S. A. Hussein, and S. A. Ahmed, J. Pharm. Biomed. Anal., 2005, 39, 139.

24. T. Pérez-Ruiz, C. Martínez-Lozano, V. Tomás, and C. Sidrach de Cardona, Talanta, 1993, 40, 1361.

25. M. Sánchez and J. J. Sánchez-Aibar, Analyst, 1996, 121, 1581.

26. M. M. Ayad, A. A. Shalaby, and H. E. Abdllatef, J. Pharm. Biomed. Anal., 1999, 20, 557.

27. R. A. S. Lapa, J. L. F. C. Lima, and J. L. M. Santos, Anal. Chim. Acta, 2000, 419, 17.

28. M. M. Ayad, H. E. Abdellatef, M. M. El-Henewee, and H. M. El-Sayed, Spectrochim. Acta, Part A, 2007, 66, 106.

29. M. A. Castillo and L. Bruzzone, Anal. Sci., 2006, 22, 431.

30. G. M. Escandar, P. C. Damiani, H. C. Goicoechea, and A. C. Olivieri, Microchem. J., 2006, 82, 29.

31. A. Muñoz de la Peña, A. Espinosa-Mansilla, M. I. Acedo Valenzuela, H. C. Goicoechea, and A. C. Olivieri, Anal. Chim. Acta, 2002, 463, 75.

32. A. Afkhami, N. Sarlak, and A. Reza Zarei, Talanta, 2007, 71,893 .

33. M. J. Culzoni, H. C. Goicoechea, G. A. Ibañez, V. A. Lozano, N. R. Marsili, A. C. Olivieri, and A. P. Pagani, Anal. Chim. Acta, 2008, 614, 46.

34. A. C. Olivieri, H. C. Goicoechea, and F. A. Iñón, Chemom. Intell. Lab. Syst., 2004, 73, 189.

35. D. M. Haaland and E. V. Thomas, Anal. Chem., 1988, 60, 1193.

36. H. C. Goicoechea and A. C. Olivieri, Trends Anal. Chem., 2000, 19, 599.

37. "British Parmacopoeia", 1998, H. M. Stationery Office, London, 206, 711.

38. H. Martens and T. Naes, "Multivariate Calibration", 1989, Wiley, Chichester.

39. J. N. Millar and J. C. Miller, "Estadística y Quimiometría para Química Analítica", 4th ed., 2002, Prentice Hall, Madrid, 45 - 51 . 